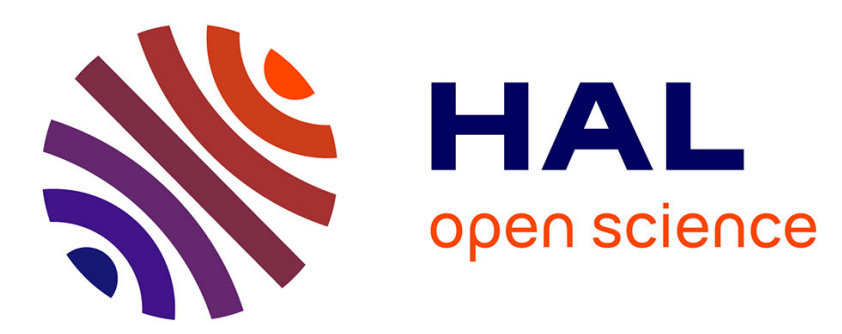

\title{
Elastic wave generated by granular impact on rough and erodible surfaces
}

\author{
Vincent Bachelet, Anne Mangeney, Julien de Rosny, Renaud Toussaint, \\ Maxime Farin
}

\section{- To cite this version:}

Vincent Bachelet, Anne Mangeney, Julien de Rosny, Renaud Toussaint, Maxime Farin. Elastic wave generated by granular impact on rough and erodible surfaces. Journal of Applied Physics, 2018, 123 (4), pp.044901. 10.1063/1.5012979 . hal-01907610

\section{HAL Id: hal-01907610 https://hal.science/hal-01907610}

Submitted on 29 Oct 2018

HAL is a multi-disciplinary open access archive for the deposit and dissemination of scientific research documents, whether they are published or not. The documents may come from teaching and research institutions in France or abroad, or from public or private research centers.
L'archive ouverte pluridisciplinaire HAL, est destinée au dépôt et à la diffusion de documents scientifiques de niveau recherche, publiés ou non, émanant des établissements d'enseignement et de recherche français ou étrangers, des laboratoires publics ou privés. 


\section{Elastic wave generated by granular impact on rough and erodible surfaces}

Vincent Bachelet, Anne Mangeney, Julien de Rosny, Renaud Toussaint, and Maxime Farin

Citation: Journal of Applied Physics 123, 044901 (2018);

View online: https://doi.org/10.1063/1.5012979

View Table of Contents: http://aip.scitation.org/toc/jap/123/4

Published by the American Institute of Physics

\section{Scilight}

Sharp, quick summaries illuminating the latest physics research 


\title{
Elastic wave generated by granular impact on rough and erodible surfaces
}

\author{
Vincent Bachelet, ${ }^{1, a)}$ Anne Mangeney, ${ }^{1,2}$ Julien de Rosny, ${ }^{3}$ Renaud Toussaint, ${ }^{4}$ \\ and Maxime Farin ${ }^{1,5}$ \\ ${ }^{1}$ Institut de Physique du Globe de Paris, Université Paris-Diderot, Sorbonne Paris Cité, CNRS (UMR 7154), \\ 75005 Paris, France \\ ${ }^{2}$ ANGE Team, Inria, Lab. J.-L. Lions, CNRS, 75005 Paris, France \\ ${ }^{3}$ Institut Langevin, ESPCI Paris, CNRS, PSL Research University, 75005 Paris, France \\ ${ }^{4}$ Institut de Physique du Globe de Strasbourg, Université de Strasbourg/EOST, CNRS, 67000 Strasbourg, France \\ ${ }^{5}$ Seismological Laboratory, California Institute of Technology, Pasadena, California 91125, USA
}

(Received 9 November 2017; accepted 2 January 2018; published online 23 January 2018)

\begin{abstract}
The elastic waves generated by impactors hitting rough and erodible surfaces are studied. For this purpose, beads of variable materials, diameters, and velocities are dropped on (i) a smooth PMMA plate, (ii) stuck glass beads on the PMMA plate to create roughness, and (iii) the rough plate covered with layers of free particles to investigate erodible beds. The Hertz model validity to describe impacts on a smooth surface is confirmed. For rough and erodible surfaces, an empirical scaling law that relates the elastic energy to the radius $R_{b}$ and normal velocity $V_{z}$ of the impactor is deduced from experimental data. In addition, the radiated elastic energy is found to decrease exponentially with respect to the bed thickness. Lastly, we show that the variability of the elastic energy among shocks increases from some percents to $70 \%$ between smooth and erodible surfaces. This work is a first step to better quantify seismic emissions of rock impacts in natural environment, in particular on unconsolidated soils. Published by AIP Publishing.
\end{abstract}

https://doi.org/10.1063/1.5012979

\section{INTRODUCTION}

Rockfalls represent major natural hazards for humans and infrastructures, ${ }^{1}$ and a better understanding of triggering mechanisms and of their dynamics is required. However, rockfalls are difficult to monitor because of their unpredictability. In this context, the seismic waves generated by rock impacts and granular flows provide a unique tool to detect, localize, and monitor these events. ${ }^{2-8}$ Indeed, the installation of seismic stations is relatively easy, and the stations may record the generated seismic signal far from the source.

The link between the rockfall properties (fall height, volume, propagation velocity, extension, etc.) and the corresponding seismic signal characteristics (signal duration and energy, frequency content, envelope properties) is, however, difficult to establish. For instance, rock impacts on the ground have been differentiated from mass flows as the spectrograms of the firsts present wide frequency and short time bands, whereas the seconds a triangular shape. ${ }^{9}$ More precisely, strong correlations between the signal duration and the run-out distance, ${ }^{10}$ or between the signal envelope and the drop height, volume and potential energy ${ }^{11}$ have been observed. Moreover, the rockfall volume has been recovered from the generated seismic energy ${ }^{12,13}$ or magnitude. ${ }^{14}$ It made possible to monitor the spatio-temporal evolution of rockfall activity and to study its link with external forcing (seismic, volcanic and rainfall activity). ${ }^{15}$ To our knowledge, there is no study trying to quantify the effect of an erodible (i.e., unconsolidated) granular bed on the seismic efficiency. However, it has been shown to change it of some orders of

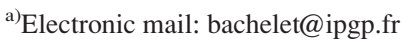

magnitude. ${ }^{6,16,17}$ To quantify this effect more precisely is very important because rockfalls could occur on beds covered by the deposit of former events or on unconsolidated soils.

Experimentally, a theoretical description of the waves generated by impacts of grains has been proposed, ${ }^{17-21}$ essentially based on the Hertz contact theory. ${ }^{22,23}$ This theory has also been used on field to quantify the sediment transport in rivers from the generated seismic signal. ${ }^{24-26}$ Scaling laws can then be established making it possible to recover the mass and velocity of the impactor from the radiated elastic energy and the mean frequency content (or frequency bandwidth) of the signal. ${ }^{17}$ The presence of an erodible bed changes the seismic efficiency because part of the impact energy is absorbed. The behavior of a granular bed impacted by a grain is a complex process. By investigating the dynamics of an intruder penetrating an unconsolidated granular bed, a scaling law between the drop height and the penetration depth has been established. ${ }^{27,28}$ The dependency between the impacting bead properties (angle of impact, velocities before and after the impact) and the properties of the ejected ones (number, vertical and horizontal ejection angle distribution) has been investigated experimentally ${ }^{29,30}$ and recovered theoretically. ${ }^{31}$ Moreover, a 2D Discrete Element Model (DEM) simulation ${ }^{32}$ has shown different dynamics of the impacting bead, depending on the radius of the beads in the granular medium. The acoustic wave propagation into a granular bed following an impact is tricky, in particular, owing to the development of complex force networks ${ }^{33,34}$ (see also, e.g., ${ }^{35}$ for a review). The situation is even more complicated on the field (ground composed of grains of variable materials, sizes and shapes, presence of 
interstitial fluid). In this context, we propose to quantify empirically the effect of a rough surface and an erodible bed on the acoustic emissions. More precisely, we aim at tuning the coefficient and powers of the scaling law obtained for a bead impact on smooth surface, to the case of rough and erodible ones.

The paper is organized as follows. The theoretical background recalling the scaling laws based on the Hertz contact law is presented in Sec. II. Then, Sec. III is devoted to the description of the experimental setup. The results are presented in Sec. IV. They are subdivided into three subsections: the evolution of (i) the averaged radiated elastic energy $W_{e l}$ and (ii) its fluctuations according to the erodible surface thickness; and (iii) the tuning, for each surface, of the $W_{e l}$ scaling law established for the smooth surface. Lastly the results are summarized in the Sec. V.

\section{THEORETICAL BACKGROUND}

To express the elastic wave generated during a bead impact on a plate as a function of the impact parameters, one has to model the contact dynamic of the falling bead. To that end, we use the non-linear Hertz contact law in the case of an elastic impact. It gives the expression of the force between the bead of radii $R_{b}$ and the surface, as a function of the bead center of mass displacement $\delta_{z}$ due to its deformation 22,23

$$
F_{z}(t)=-K \delta_{z}^{3 / 2}(t)
$$

with

$$
K=\frac{4}{3} \sqrt{R_{b}} E^{*}
$$

The effective Young modulus $E^{*}$ of the system is given by

$$
\frac{1}{E^{*}}=\frac{1-\nu_{b}^{2}}{E_{b}}+\frac{1-\nu_{s}^{2}}{E_{s}} .
$$

The letters $E_{b}$ and $\nu_{b}$ (respectively, $E_{s}$ and $\nu_{s}$ ) correspond to the Young modulus and Poisson coefficient of the bead (respectively of the surface). This relationship still holds when the impacted surface has a local curvature radius $R_{s}$. In this case, $R_{b}$ become $R^{*}$ such as $1 / R^{*}=1 / R_{b}+1 / R_{s}$.

The time dependence of the interpenetration $\delta_{z}$ of a bead of mass $m$, impacting a plate with an initial speed $V_{z}$, is the solution of Newton's law. For convenience, we handle the dimensionless interpenetration distance $\delta_{z}^{*}\left(\delta_{z}^{*}=\delta_{z} / D\right)$ and dimensionless time $t^{*}\left(t^{*}=t / T\right)$ where $D$ and $T$ are given by $m^{2 / 5} V_{z}^{4 / 5} / K^{2 / 5}$ and $D / V_{z}$, respectively. The dimensionless equation of motion is then

$$
\frac{d^{2} \delta_{z}^{*}\left(t^{*}\right)}{d t^{* 2}}=-\delta_{z}^{* 3 / 2}\left(t^{*}\right) .
$$

The general expression of the elastic energy generated during an impact is provided by the mechanical work $W_{e l}^{H e r t z}=\int_{-\infty}^{\infty}|F(t)|^{2} Y_{e l} \mathrm{~d} t$ where $Y_{e l}$ is the admittance of the surface. For plates, it is independent of the frequency: $Y_{e l}=1 /\left(8 \sqrt{B \rho_{p} h_{p}}\right)$, with $B=\left(h_{p}^{3} E_{p}\right) /\left(12\left(1-\nu_{p}^{2}\right)\right)$ the bending stiffness and $h_{p}, E_{p}$, and $\nu_{p}$ the thickness, Young modulus, and Poisson coefficient of the plate, respectively. ${ }^{17,36,37}$ For practicality, it is possible to compute first the dimensionless work $W_{e l}^{*}$ which depends on the dimensionless force $F^{*}: W_{e l}^{*}=\int_{-\infty}^{\infty}\left|F^{*}\left(t^{*}\right)\right|^{2} \mathrm{~d} t^{*} \approx 1.73$. The benefit of this procedure is to solve the problem once for all because the actual elastic energy is linearly dependent of the dimensionless one, which the prefactor embeds all the parameters of the experiment. The same procedure is possible for the mean frequency of the radiated waves by the impact: $f_{\text {mean }}^{\text {Hertz }}$ $=f_{\text {mean }}^{*} / T$ with $f_{\text {mean }}^{*}=\left(\int_{0}^{\infty}\left|\tilde{A}_{z}^{*}\left(f^{*}\right)\right| f^{*} \mathrm{~d} f^{*}\right) /\left(\int_{0}^{\infty}\left|\tilde{A}_{z}^{*}\left(f^{*}\right)\right| \mathrm{d} f^{*}\right)$ $\approx 0.45$. Finally, the scaling laws computed by Farin et al. ${ }^{17}$ are recovered

$$
\begin{aligned}
W_{e l}^{\text {Hertz }} & =a_{0} R_{b}^{5} V_{z}^{11 / 5}, \\
f_{\text {mean }}^{\text {Hertz }} & =a_{0}^{\prime} R_{b}^{-1} V_{z}^{1 / 5},
\end{aligned}
$$

with

$$
\begin{aligned}
& a_{0}=\left(\frac{4}{3}\right)^{2} \pi^{8 / 5} Y_{e l}\left(E^{*} \rho^{4}\right)^{2 / 5}\left(\frac{R_{s}}{R_{b}+R_{s}}\right)^{1 / 5} W_{e l}^{*}, \\
& a_{0}^{\prime}=\frac{1}{\pi^{2 / 5}}\left(\frac{E^{*}}{\rho}\right)^{2 / 5}\left(\frac{R_{s}}{R_{b}+R_{s}}\right)^{1 / 5} f_{\text {mean }}^{*} .
\end{aligned}
$$

The convergence of $W_{e l}^{\text {Hertz }}$ and $f_{\text {mean }}^{\text {Hertz }}$ according to the frequency content is investigated (Fig. 1). The energy converges much faster than the mean frequency and reaches 99\% of its value when $70 \%$ of the frequencies of the first lobe is taken into account. For the same spectral content, $f_{\text {mean }}^{\text {Hertz }}$ reaches only $73 \%$ of its value. We will see in Sec. III that the frequency content of the impacts can be as high as twice of the bandwidth of the sensors measuring it. Thus, the radiated elastic energy is the only reliable quantity. That is why the mean frequency will not be considered in this paper.

\section{EXPERIMENTAL SETUP}

Beads of various diameters and densities are dropped on several surfaces from variable heights $H$, i.e., variable impact

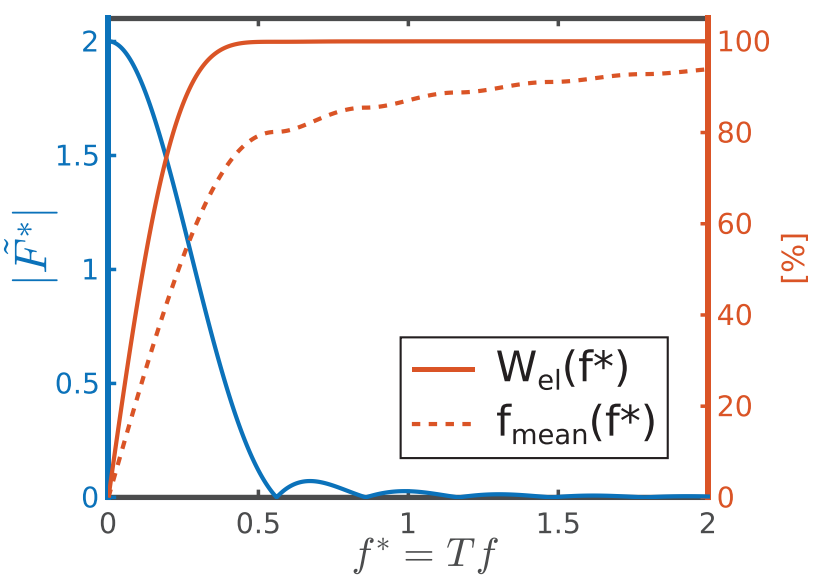

FIG. 1. Spectrum of the dimensionless force (blue). The relative estimation of the radiated elastic energy (red line) and mean frequency (dashed red line) when they are estimated only using the frequency components between 0 and $f$. They are normalized by the real values computed when the entire spectrum is taken. 
TABLE I. Elastic parameters.

\begin{tabular}{lccc}
\hline \hline Material & $\rho\left(\mathrm{kg} / \mathrm{m}^{3}\right)$ & $E(\mathrm{GPa})$ & $\nu$ (Poisson coefficient) \\
\hline PMMA & 1180 & 4.4 & 0.37 \\
Steel & 7800 & 220 & 0.30 \\
Glass & 2500 & 74 & 0.40 \\
\hline \hline
\end{tabular}

speed $V_{z}=\sqrt{2 g H}$. Table I presents the elastic parameters of the materials, and Table II summarizes the experimental conditions. The dropped beads are composed of two materials: steel and glass. Diameters range between 2 and $5 \mathrm{~mm}$ $( \pm 1 \mu \mathrm{m})$ for the steel beads and 2 to $10 \mathrm{~mm}( \pm 0.25 \mathrm{~mm})$ for the glass ones. They are dropped without initial velocity or initial rotation, thanks to a mechanical diaphragm from 10 , 17 , and $30 \mathrm{~cm}$ heights. The impacted surfaces are of three different types (Fig. 2): (i) a smooth PMMA 1-cm thick plate $(1 \mathrm{~m} \times 1 \mathrm{~m})$, (ii) stuck glass beads on the PMMA plate to create roughness, and (iii) the rough plate covered with layers of free particles to investigate erodible beds. Surfaces (ii) and (iii) are made using either $2 \mathrm{~mm}$ or $3 \mathrm{~mm}$ glass beads. The beads composing the rough bed are glued on the plate with phenyl salicylate, a crystalline substance with low fusion temperature. The surface packing density $\phi_{2 D}$ of the glued beads is estimated using image processing which leads to the value of $\phi_{2 D}=0.90$. It is very close to the maximum value $\pi /(2 \sqrt{3}) \approx 0.91$ corresponding to a hexagonal packing, which may be explained by beads overlapping since the rough surface is not exactly one-bead thick. The erodible surfaces are prepared by pouring the free beads on top of the rough surface and by removing the excess with a squeegee. After each drop, the surface is recreated with the same procedure. The volume packing fraction of the erodible layers is estimated to be $\phi_{3 D}=0.56 \pm 0.4$ by weighing a sample of the bed whose volume is known. The variability of its value is an advantage in this study because we want to quantify its effect on acoustic measurements.

The elastic waves generated during the impacts are recorded with 8 accelerometers (type 8309 , charge accelerometers, Bruel and Kjaer, bandwidth $10 \mathrm{~Hz}-54 \mathrm{kHz}$ ) stuck on the backside of the plate and around the impact zone (Fig. 3 ). Within this frequency range, the generated waves are essentially $A_{0}$ modes of Lamb waves. ${ }^{21}$ In order to explore the intrinsic variability of the initial conditions, each drop is

TABLE II. Experimental parameters. $e^{*}$ corresponds to the bed thickness normalized by the diameter of the beads composing it (either 2 or $3 \mathrm{~mm}$ glass beads).

\begin{tabular}{lccc}
\hline \hline Impacted surface & \multicolumn{1}{c}{$d^{\mathrm{a}}$} & $H^{\mathrm{b}}$ & $V_{z}^{\mathrm{c}}$ \\
\hline Smooth $\left(e^{*}=0\right)$ & Steel: $[3.175,4,4.5,5]$ & 10 & 1.4 \\
Rough $\left(e^{*}=1\right)$ & Glass: $[3,4,5,10]$ & 17 & 1.8 \\
Erodible $e^{*}=2.5$ & & 30 & 2.4 \\
Erodible $e^{*}=5$ & & \\
Erodible $e^{*}=10$ & & & \\
\hline \hline
\end{tabular}

${ }^{\mathrm{a}}$ Drop bead diameter $(\mathrm{mm})$.

${ }^{\mathrm{b}}$ Drop height $(\mathrm{cm})$.

${ }^{\mathrm{c}}$ Impact velocity $\left(\mathrm{ms}^{-1}\right)$.
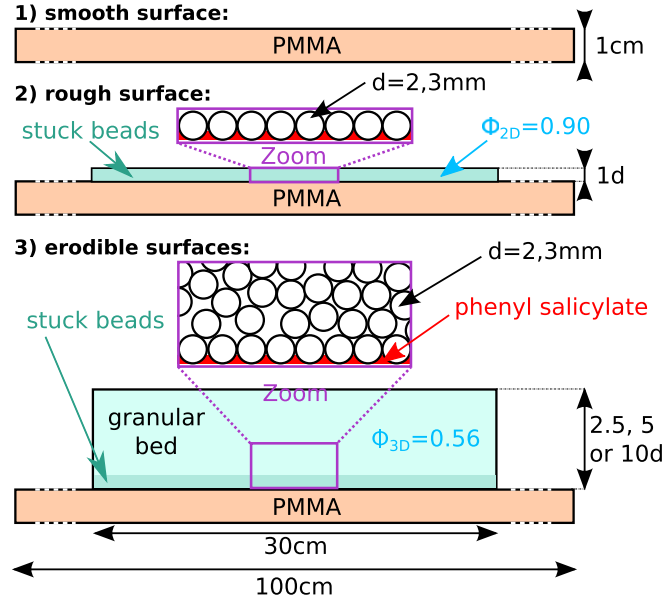

FIG. 2. Sketch of the three different impacted surfaces: smooth, rough, and erodible.

performed 5 times. On the rough surface, beads are dropped 50 times and monitored with two cameras (Optronis CamRecord CR600x2 and MotionBLITZ EoSens Cube7, both at $500 \mathrm{fps}$ ) to investigate the effects of the impactor bounce angle.

It is possible to extend the scaling laws for a smooth plate [formula (5)] to the case of the rough impacted surface made of stuck beads. Assuming a perfect hold of the beads (no change in contact dynamic and perfect energy transmission from the fixed beads to the plate), the formula (6) can be left as it: $R_{s}$ becomes the radius of the gluing beads and $E^{*}$ holds their elastic parameters. Furthermore, $R_{s} /\left(R_{b}+R_{s}\right)$ can be assumed constant and equal to 0.8 because range of radius $R_{b}$ investigated lies between $R_{s}$ and $2.5 R_{s}$. However, neither model nor scaling laws has been yet proposed to connect source properties and generated waves for impacts on erodible surfaces (free beads poured on the glued ones). One

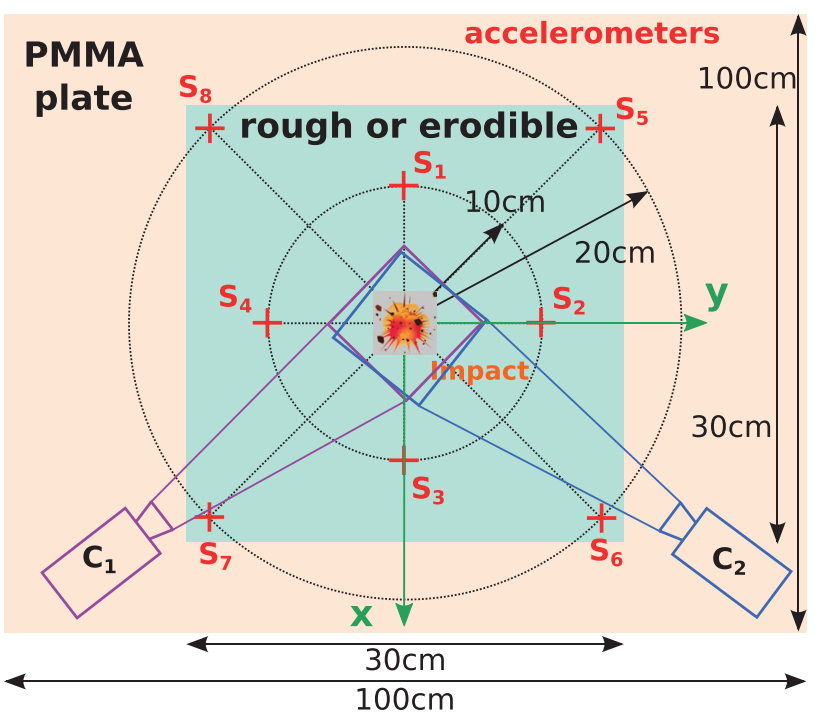

FIG. 3. Sketch of the experimental setup. The red crosses indicate the positions of the sensors $S_{i}$, located at about $10 \mathrm{~cm}$ and $20 \mathrm{~cm}$ from the impact location. Impacts on the rough surfaces are additionally filmed using two cameras $C_{1}$ and $C_{2}$. 
objective of the paper is to assess the scaling laws able experimentally to reproduce the observations in that case.

We measure the elastic energy $W_{e l}$ of the vibrations emitted by the impactor strikes using the energy flux conservation of the first wave arrivals ${ }^{21}$

$$
W_{e l}=2 r h_{p} \rho_{p} \int_{0}^{\infty} v_{g}(\omega)\left|\tilde{v}_{z}(r, \omega)\right|^{2} e^{\gamma(\omega) r} \mathrm{~d} \omega .
$$

It depends on the source-receiver distance $r$, thickness $h_{p}$ and density $\rho_{p}$ of the plate, viscous attenuation coefficient $\gamma(\omega)$ with $\omega$ the angular frequency, group velocity $v_{g}(\omega)$, and vertical displacement velocity of the wave in the Fourier space $\tilde{v}_{z}(r, \omega)$. The first wave arrivals are selected to avoid any reflection at the boundaries of the plate that could lead to overestimation of the radiated elastic energy (Fig. 4). The factor $\exp (\gamma(\omega) r)$ compensates absorption losses during wave propagation in the plate. Absorption rate $\gamma(\omega)$ is sensitive to the load exerted on the plate ${ }^{38,39}$ and depends on the weight of the beads forming the bed. We measure it for each bed thickness throughout additional experiments by measuring the response of the plate between transducers at various distances (Fig. 5). At low frequencies, the group velocity of the elastic wave (mode A0) is well approximated by ${ }^{21,40}$

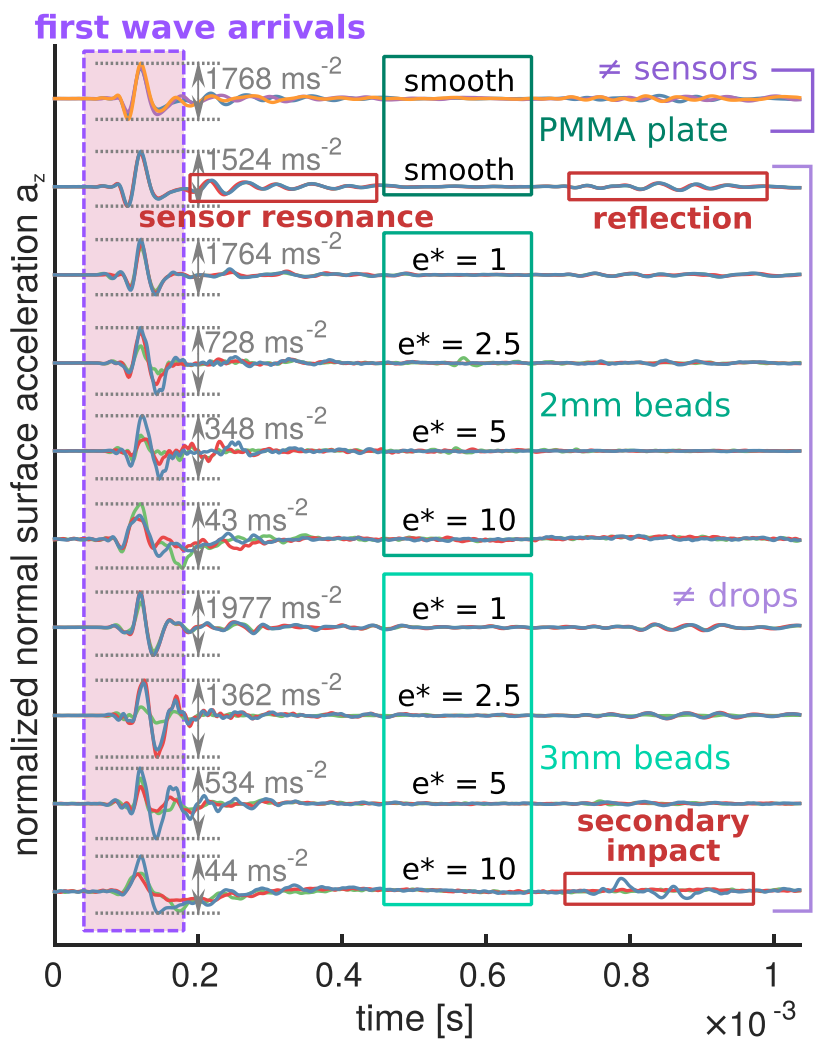

FIG. 4. Normal accelerations of the elastic wave generated by a $5 \mathrm{~mm}$ diameter glass bead impacting the different surfaces at the velocity $V_{z}=1.8 \mathrm{~ms}^{-1}$. The distance between the receivers and the impact location is equal to $10 \pm 1 \mathrm{~cm}$. On the top plot, four signals recorded by four accelerometers are shown, whereas the different signals on the other plots correspond to different drops for one sensor, for the smooth plate, and on an erodible bed of different normalized thicknesses $e^{*}$. Sensor resonance and plate boundary reflection are clearly visible on the smooth surface signals. The wave generated by one ejected bead is discernible on the last signal ("secondary impact").

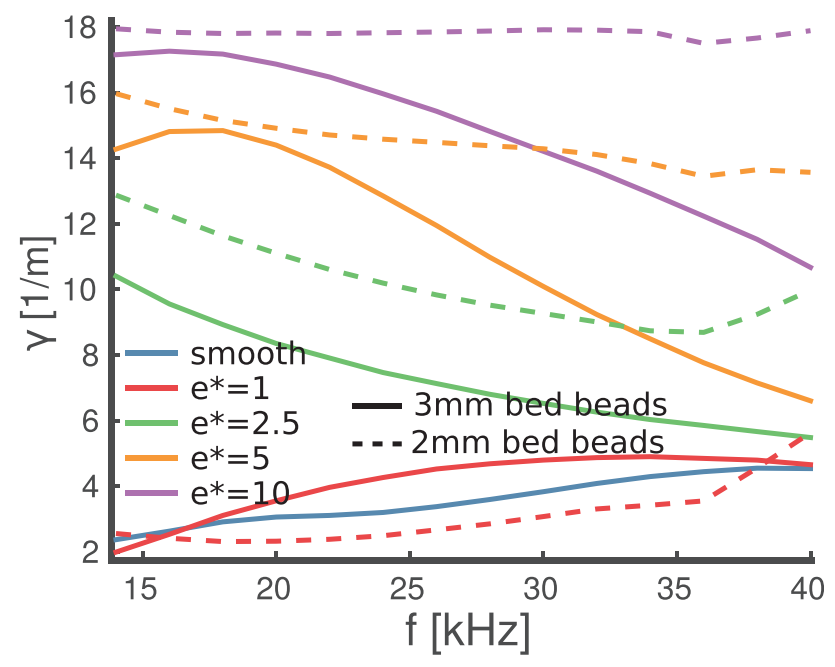

FIG. 5. Viscous attenuation coefficient $\gamma$ of the PMMA plate for different bed thicknesses $e^{*}\left(u=u_{0} / \sqrt{r} \exp \left(-\frac{\gamma}{2} r\right)\right.$, with $u$ the wave amplitude, $r$ the source-receiver distance, and $u_{0}$ a coefficient).

$$
v_{g}(\omega)=2 k \sqrt{\frac{B}{\rho_{p} h_{p}}},
$$

with $\omega$ the pulsation, $k$ the wavenumber, and $B$ the bending stiffness. The source-sensor distance $r$ is recovered throughout a beam-forming processing (Fig. 6). Here we cannot apply classical beam-forming based on time delays because of the wave dispersion. To overcome this limitation, each frequency of the recorded signals is back propagated with the corresponding phase velocity. In addition, better localization is recovered by considering only phases of the signals in the $2-20 \mathrm{kHz}$ frequency bandwidth because attenuation is low enough to be neglected.

The bounce angle of a particle on the rough surface, i.e., the angle between the vertical and bead direction just after
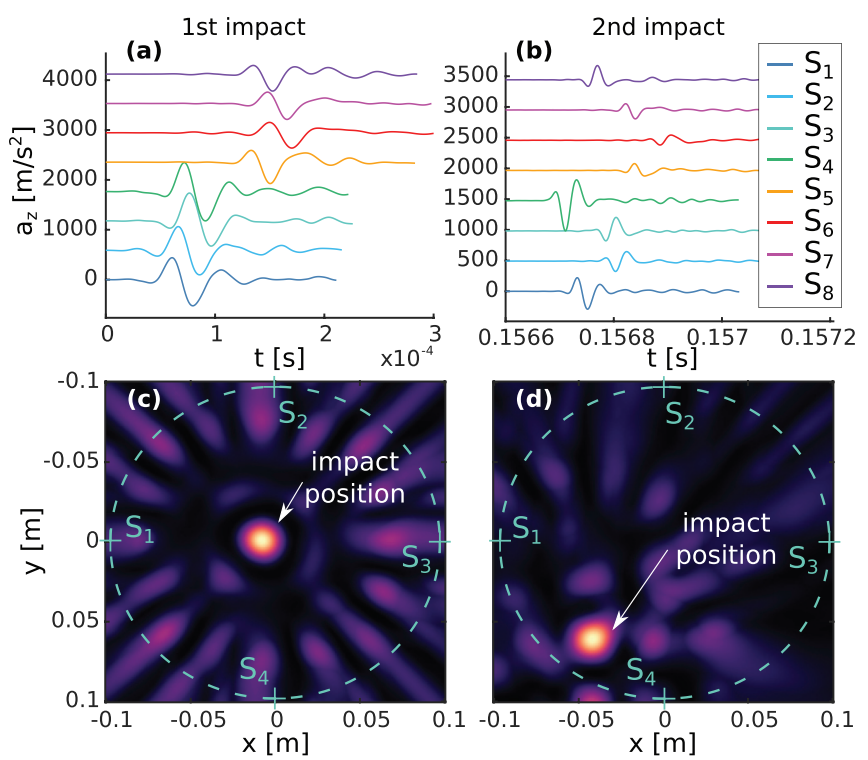

FIG. 6. Localization of the first two impact positions of a $5 \mathrm{~mm}$ glass bead on the rough surface using beam-forming with 8 accelerometers. Raw acoustic signals (a) and (b) are back propagated to various test source positions (c) and (d). The color scale corresponds to the criterion $\mid \max _{t}\left(a_{\text {stack }}(t)\right)$ $* \min _{t}\left(a_{\text {stack }}(t)\right) \mid, a_{\text {stack }}$ being the stack of the 8 back-propagated signals. 
the impact, is measured. It is important because it drives the radiated elastic energy. Two independent methods are used. The first one is based on the recording of the stereoscopic view using 2 fast video cameras (Fig. 7). To begin with, the two $2 \mathrm{D}$ bead trajectories are recovered on each camera, and they are mixed to provide the $3 \mathrm{D}$ trajectory. The second method uses the acoustic beam-forming localization of the first and second impacts: knowing the distance and time between the two impacts, the free fall parabola of the trajectory and then the bounce angle are deduced. The accuracy of the two methods are similar with, respectively $\pm 4^{\circ}$ and $\pm 3^{\circ}$, but the first one is more robust. Indeed, the acoustic method fails when the impact position (after bounce) is outside the area lying between accelerometers.

\section{RESULTS}

On rough and erodible surfaces, the bead impact is a non-deterministic process, i.e., the radiated elastic energy resulting from two successive bead impacts is different. So, a statistical analysis should be performed. First, we quantify energy conversion, from kinetic to elastic. Second, we analyze the dispersion of the radiated elastic energy. Third, we
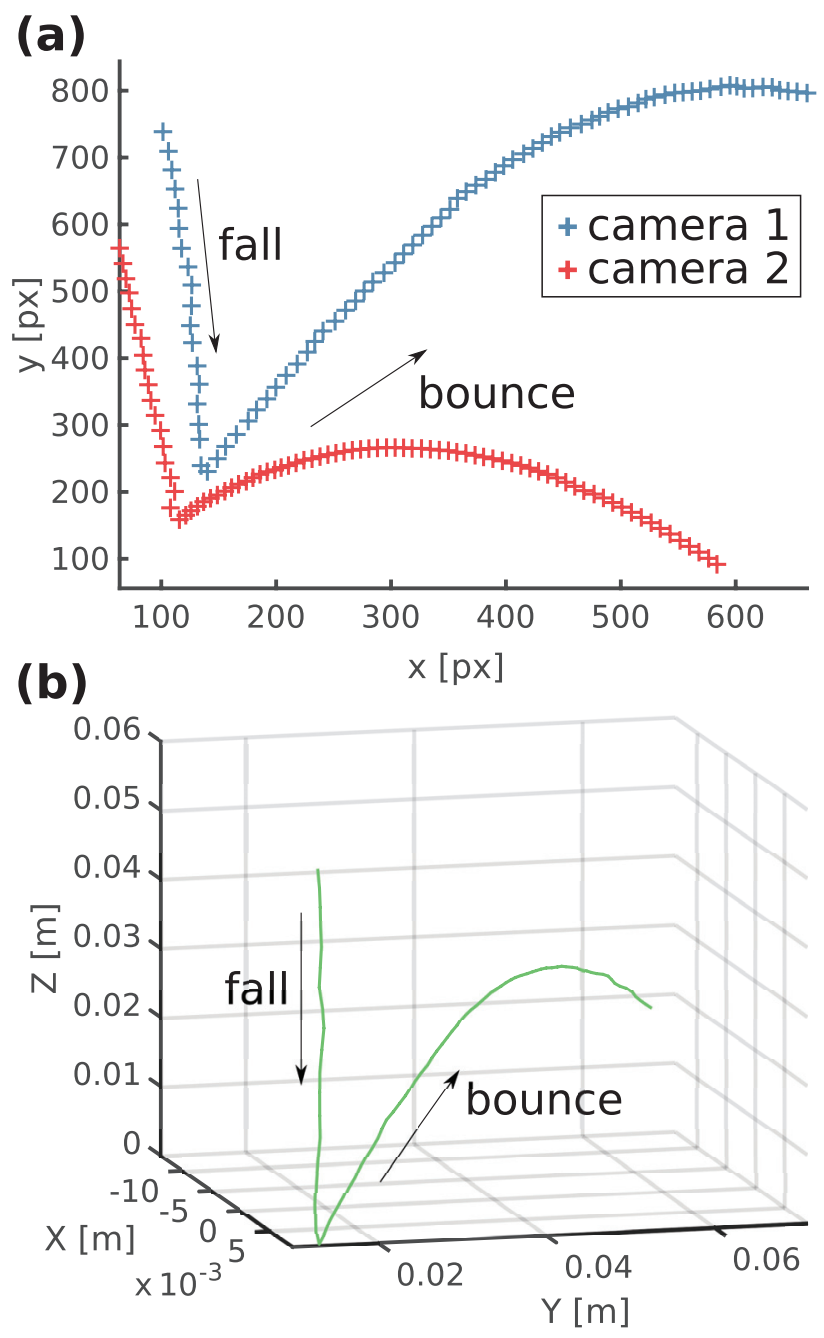

FIG. 7. Stereoscopy processing: combination of two 2D trajectories recorded by 2 fast video cameras (a) in order to obtain the corresponding 3D trajectory (b). propose tuned scaling laws for rough and erodible surfaces. Obviously, discrepancies are observed from the theoretical law established for smooth surfaces.

\section{A. Energy attenuation due to rough and erodible surfaces}

The conversion from kinetic energy at the moment of impact $E_{c}^{\text {impact }}=0.5 m V_{z}^{2}$ to elastic energy $W_{e l}$ for the various surfaces is presented in Fig. 8. A strong decrease of $\sim 2$ orders of magnitude is visible between the smooth surface and the thickest bed. To go further, the ratio between $W_{e l}$ and $E_{c}^{\text {impact }}$ is presented in function of the bed thickness $e^{*}$ (Fig. 9). The decay factor 100 between the smooth and the thickest erodible surface is recovered. The energy conversion is about twice more efficient for the largest beads $(10 \mathrm{~mm}$ diameter compared to $3 \mathrm{~mm}$ ones) for all surfaces. Steel beads also convert twice more energy than the glass ones for the smooth and rough surfaces. For the erodible surface, the fluctuations are too large to observe significant difference between steel and glass beads. When the bed thickness lies between 2 and 6 , the relative radiated energy is larger when the impactor hits beds made of $3 \mathrm{~mm}$-radius beads instead of $2 \mathrm{~mm}$-radius ones. For the rough and thick bed, no clear trend is observed. Finally, a global fit of the form $W_{e l} / E_{c}^{i m p a c t}=a e^{-b e^{*}}$ has been performed over all the data (Fig. 9): $a=0.13$ quantifies the average energy conversion for the smooth surface, and $b \approx 0.5$ the exponential decay according to the bed thickness.

On field, acoustic emissions of debris flows on a rough and an erodible surface have been recorded. ${ }^{16}$ A loss of factor 100 of the signal energy has been measured. Knowing the thickness of the erodible surface $(0.34 \mathrm{~m})$, it is possible to invert the exponential decay law to have an estimation of the bed particles diameter: $0.34 \mathrm{~m}$. It is close to the 84 th percentile of the distribution of the grains, which is equal to $3.1 \mathrm{~cm},{ }^{41}$ and in agreement with the fact that most of the

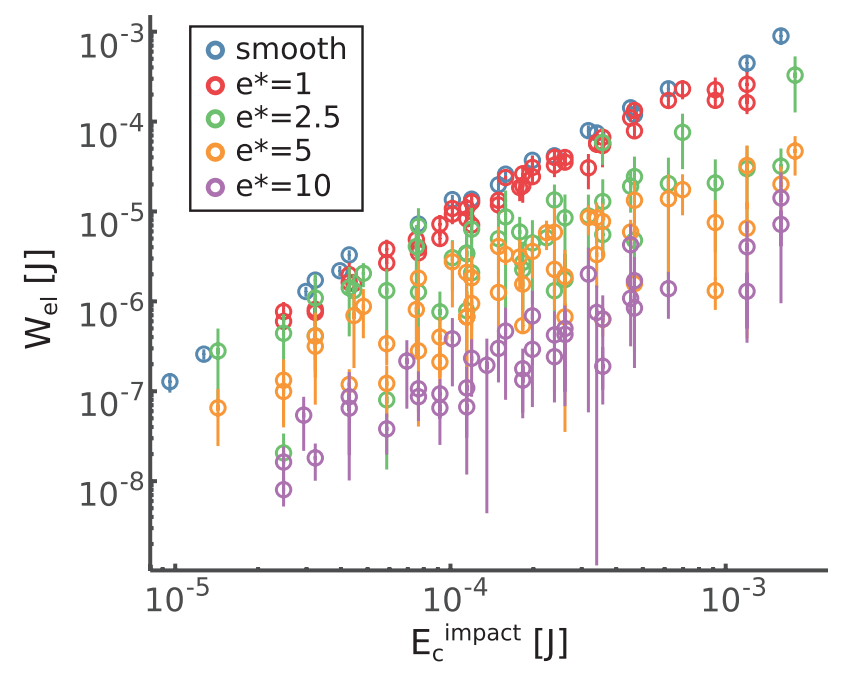

FIG. 8. Radiated elastic energy versus kinetic energy of beads at the time of impact for the different surfaces impacted. No differentiation has been done between steel and glass beads nor between diameters of beads constituting the erodible surfaces. Each point corresponds to the average value between sensors and drops, with error bars computed from the standard deviation. 


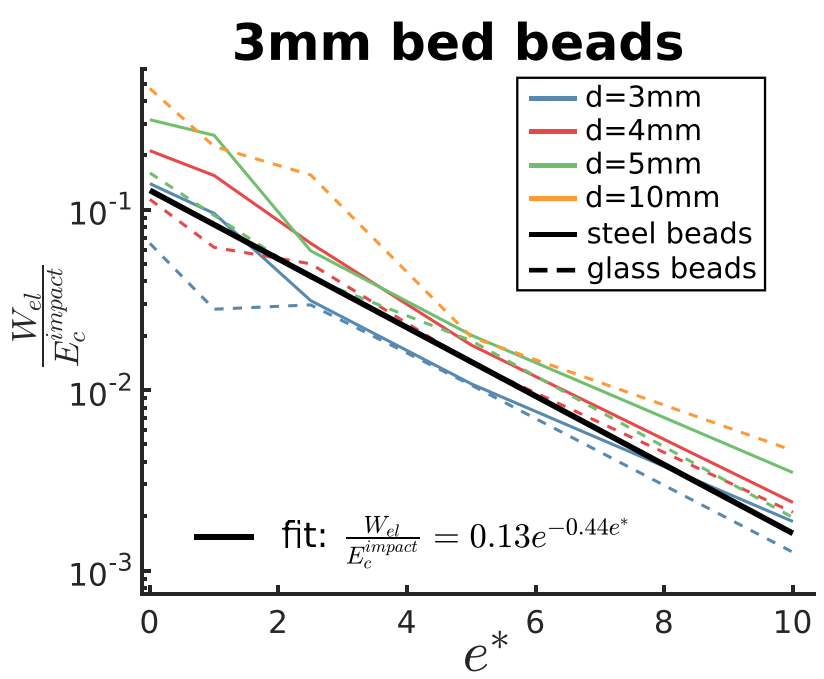

$\mathbf{2 m m}$ bed beads

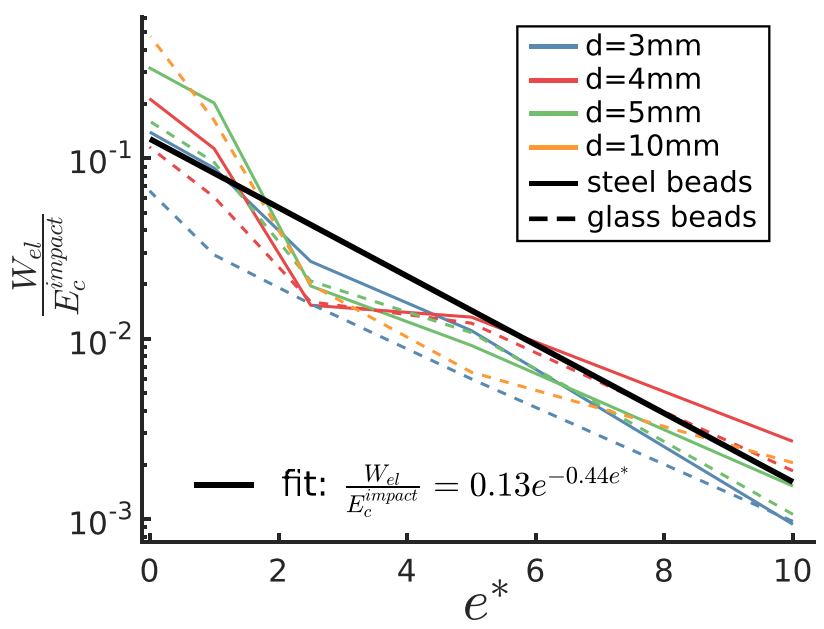

FIG. 9. Ratio between elastic and kinetic energy at the time of impact, as a function of the bed thickness $e^{*}$. Each point corresponds to the average value computed for variable $V_{z}$, sensors, and drop repetitions. The fit is established for all impacts, but impacts on the beds, constituted of $2 \mathrm{~mm}$ and $3 \mathrm{~mm}$ glass beads, are differentiated for clarity.

acoustic waves seem to be generated by the biggest particles. $^{24,26}$

\section{B. Fluctuations of the recorded elastic energy}

Our second interest is to quantify the fluctuations of $W_{e l}$ measurements induced by the different surfaces (Fig. 10). Fluctuations have two main origins: variations between similar drops for one sensor that we call "impact fluctuations" and mismatch between sensors for one drop called "sensor fluctuations". The impact fluctuations are associated with variability in the initial experimental conditions, whereas the sensor fluctuations correspond to the mismatches between the different estimations of $W_{e l}$.

A first global observation is that the fluctuations weakly depend on the bead diameter of the bed or the impacting bead material. Actually, the difference of bead diameter (2 and $3 \mathrm{~mm}$ ) is likely too small to observe a significant deviation. The independence with respect to the bead material for erodible surfaces $\left(e^{*}>2\right)$ is more surprising because the

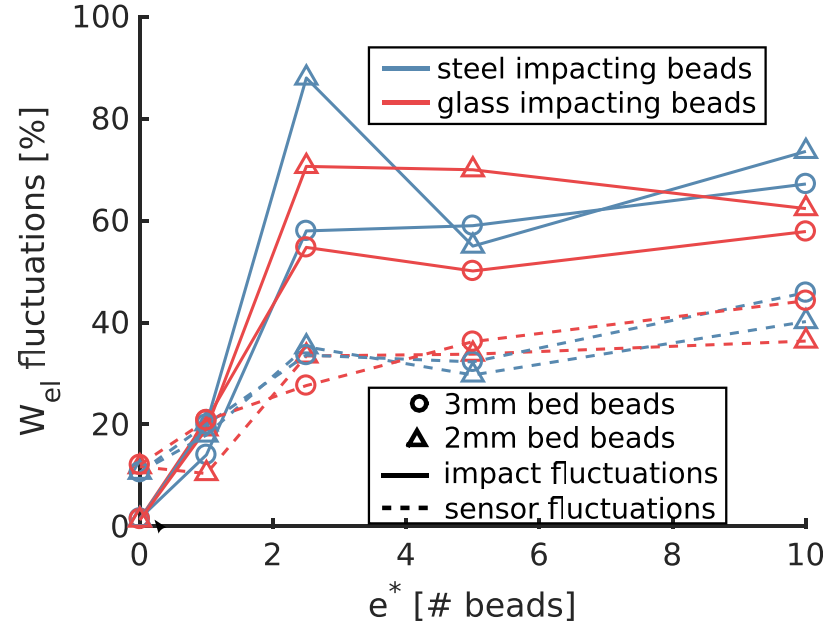

FIG. 10. Comparison of the two types of fluctuations of the elastic energy. The averaged values only are presented. The impact fluctuations are computed first by estimating the fluctuation between drops for a given sensor, impacting bead radius and velocity, and then by averaging all the values coming from all the different sensors, bead radius, and velocities (see Table II).

steel density is about three times larger than the glass one. It seems to show that an erodible surface efficiently absorbs the excessive amount of kinetic energy of the steel bead preventing a complete reorganization of the bed and strong fluctuations.

Considering the smooth surface, $W_{e l}$ fluctuations originate mainly from the sensors mismatch compared to the impact variations (10\% compared to $1 \%)$. Impact variations are only due here to variability in the initial impact positions and velocities of the dropped bead. It confirms that drops are reproducible and the accuracy of the measurements is limited by the sensors. The reasons for the mismatch between sensors may be various: inaccurate estimation of source-sensor distances, inaccurate attenuation coefficient, or imperfect control of the sensor gluing fixations.

Considering the erodible surfaces, the impact fluctuations jump from $\sim 1 \%$ to $\sim 70 \%$ between the smooth and erodible surfaces. As the bed is renewed after each drop, it is due to the different bead configurations of the bed. Each configuration has a different impact local zone, which changes the wave generation, and a different force chain network, which in turn changes the wave propagation. The fact that the impact fluctuations seem not to vary according to the bed thickness suggests that the local impact zone has a stronger influence than the propagation afterwards. It is confirmed by the fact that impact fluctuations are higher than sensor fluctuations, by a factor $\sim 2$. The second observation is that the sensor fluctuations are also higher for the erodible surfaces than for the smooth surface, by a factor of 4 . Actually, the thicker the erodible bed, the stronger the elastic coupling between the bed and the PMMA plate. As a consequence, the simple thin plate model is less and less valid (higher viscous attenuation coefficients, celerity and attenuation anisotropy,...) (see Fig. 5).

The last observation is that the main source of fluctuations is different for erodible beds than for smooth surfaces. Indeed, while sensor fluctuations are more important than impact fluctuations on smooth surfaces, it is the contrary for 
erodible beds. This shows that the effect of the potential anisotropy of the media (i.e., erodible bed) on wave propagation is smaller than the source variability.

So far, no comment has been done for the rough surface case fluctuations. A connection with the bounce angle $\theta$ of the impacting bead is highlighted here. For this purpose, additional experiments are performed, dropping glass beads of diameter $3,4,5$, and $10 \mathrm{~mm}$ fifty times, each on the rough surface made of stuck glass beads of $3 \mathrm{~mm}$ diameter. Contrary to the previous case, drops are performed at random positions to investigate a larger range of angles.

A very simple model to predict the elastic energy dependency according to the bounce angle is developed (Fig. 11)

$$
W_{e l}(\theta)=\int_{-\infty}^{\infty}\left|F_{z}(t)\right|^{2} Y_{e l} \mathrm{~d} t=\cos ^{2}\left(\frac{\theta}{2}\right) \int_{-\infty}^{\infty}\left|F_{n}(t)\right|^{2} Y_{e l} \mathrm{~d} t
$$

$F_{z}$ represents the vertical projection of the oblique force $F_{n}$ : $F_{z}=\cos (\theta / 2) F_{n}(t)$. It is due to the fact that accelerometers are only sensitive to one component. The remaining integral is given by Eq. (5)

$$
\int_{-\infty}^{\infty}\left|F_{n}(t)\right|^{2} Y_{e l} d t \hat{=} a_{0} R_{b}^{5} V_{n}^{11 / 5},
$$

where $V_{n}$ corresponds to the velocity of the impacting bead normal to the impacted bead: $V_{n}=V_{z} \cos (\theta / 2)$. Thus, combining Eqs. (5), (9), and (10) leads to

$$
W_{e l}(\theta)=\cos ^{21 / 5}\left(\frac{\theta}{2}\right) W_{e l}^{H e r t z} .
$$

The model is based on the assumptions that no friction occurs during the impact, and force is perfectly transmitted to the plate. Relatively good agreement is found with the experimental data (see Fig. 12), with an Rsquare $R^{2}$ of the fits higher than 0.7 for the $3-4-5 \mathrm{~mm}$ diameter beads. The agreement is much lower for the $10 \mathrm{~mm}$ beads $\left(R^{2}=0.34\right)$, probably because of the weight of the beads which damages the surface, in various ways according to the angle bounce. Finally, a frictionless Hertz model seems

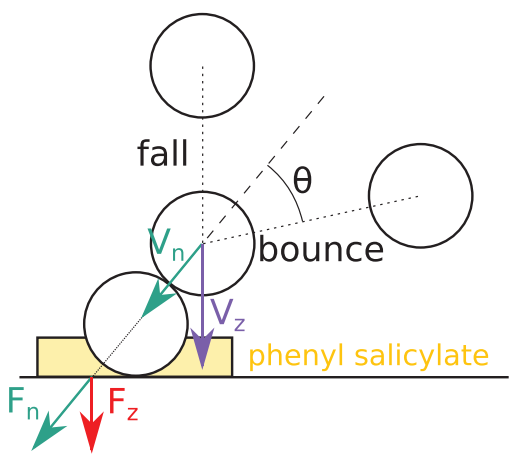

FIG. 11. Picture of the transmission of the force generated by an oblique impact on the rough surface: a bead impacts another one stuck on the plate with an angle $\theta$. The normal part $V_{n}$ only of the impact velocity $V_{z}$ is transferred to the stuck bead and generates a force $F_{n}$. This force is assumed to be totally transmitted to the plate throughout phenyl salicylate. Finally, only the vertical component $F_{z}$ of the force $F^{\prime}$ is measured.
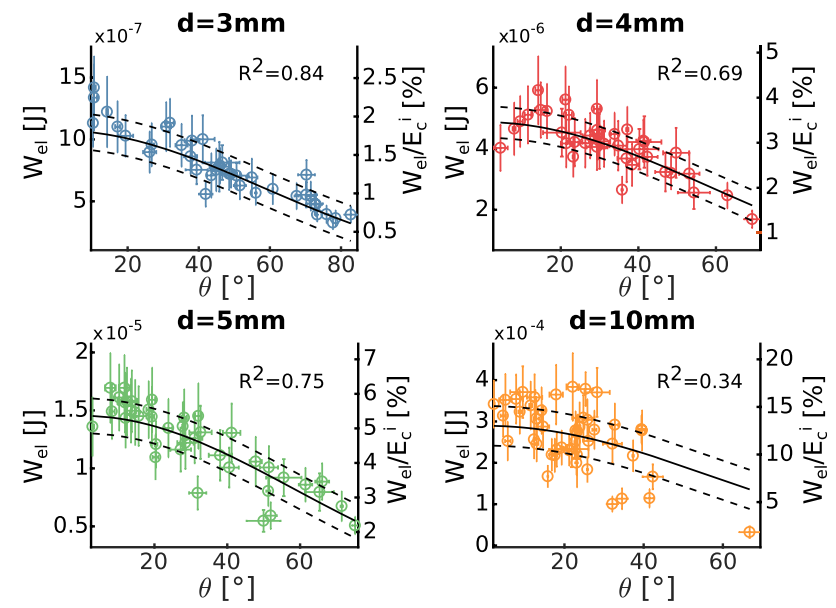

FIG. 12. Elastic energy according to the bounce angle for variable diameter glass beads falling onto the $3 \mathrm{~mm}$ rough surface. Full lines correspond to the model presented in Eq. (11) with $W_{e l}^{\text {Hertz }}$ as a fit parameter and dashed lines to the $50 \%$ confident intervals. Each point corresponds to a drop in the surface at a random position. Error bars are obtained from the standard deviations between measurements: two measurements for $\theta$ (one from the accelerometers and one from the cameras) and eight for $W_{e l}$ (one per accelerometer).

sufficient to describe the energy radiated by collisions on rough surfaces if the impact bead sizes are similar to the ones constituting the surface.

\section{Effect of bed state on the scaling laws for the radiated elastic energy}

The coefficients of the scaling law

$$
W_{e l}=W_{e l}^{0}\left(\frac{R_{b}}{R^{0}}\right)^{\alpha}\left(\frac{V_{z}}{V_{z}^{0}}\right)^{\beta},
$$

with constants $R^{0}=1 \mathrm{~m}$ and $V_{z}^{0}=1 \mathrm{~ms}^{-1}$, are estimated from linear fits in log-log scale Fig. 13. The prefactor $W_{e l}^{0}$ has the same numerical value as $a_{0}$ in Eq. (5) but is defined such that its dimension corresponds to the energy of a sphere of radius $R_{b}=1 \mathrm{~m}$ with velocity $V_{z}=1 \mathrm{~ms}^{-1}$.

Fits are very good $\left(R^{2}>0.90\right)$ for the smooth surface. The corresponding values of the coefficients are consistent with the Hertz model: $\alpha=5 \pm 0.02\left(\alpha_{\text {Hertz }}=5\right), \beta=2.25 \pm 0.05$ $\left(\beta_{\text {Hertz }}=2.2\right)$ and the experimental prefactor $W_{\text {elsteel }}^{0}$ $=510^{8} \pm 110^{8} \mathrm{~J}\left(W_{\text {el.steel }}^{0, \text { Hertz }}=4.310^{8} \mathrm{~J}\right)$ and $W_{\text {el,glass }}^{0}=510^{7}$ $\pm 110^{7} \mathrm{~J}\left(W_{\text {el,glass }}^{0, \text { Hertz }}=6.910^{7} \mathrm{~J}\right)$.

Fits are good for the rough and erodible surfaces $\left(R^{2}>0.5\right)$. The prefactor $W_{e l}^{0}$ decreases rapidly as the thickness increases, from $10^{7}-10^{8} \mathrm{~J}$ to $1-10^{4} \mathrm{~J}$. But it is expected to increase locally from the smooth to the rough surface as the effective young modulus of the impacted surface increases when passing from PMMA to glass [see formula (6) and Table I]. It is indeed the case for the steel beads but not for the glass ones. An explanation could be that the second ones excite more frequencies higher than the sensors' maximum frequency: $f_{\text {mean }}^{\text {Hertz }} \sim 40 \mathrm{kHz}$ for glass beads and $f_{\text {mean }}^{\text {Hertz }} \sim 30 \mathrm{kHz}$ for steel beads [formula (5)].

The $\alpha$ coefficient decreases from 5 to 3-4, meaning that as the bed thickness $e^{*}$ increases, the elastic energy depends less on the impacting bead radius $R_{b}$. A possible explanation 

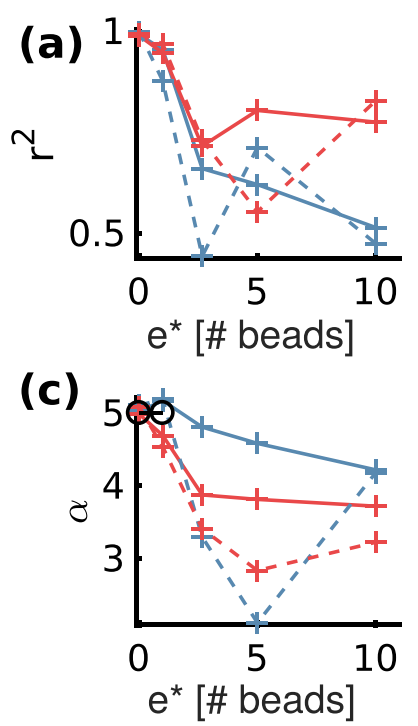

(d)
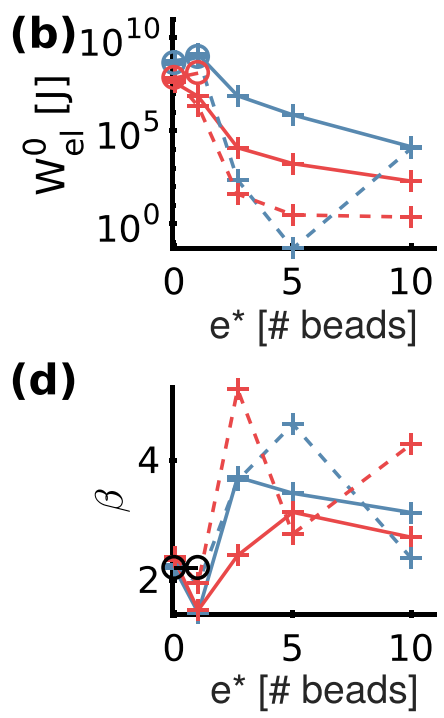

O Hertz model — steel beads $-3 \mathrm{~mm}$ bed beads + experiment $\longrightarrow$ glass beads $\ldots-2 \mathrm{~mm}$ bed beads

FIG. 13. Scaling law parameters of the elastic energy $W_{e l}=W_{e l}^{0} R_{b}^{\alpha} V_{z}^{\beta}$ fits according to the normalized bed thickness: Rsquare of the fits (a), prefactor $W_{e l}^{0}(\mathrm{~b}), \alpha(\mathrm{c})$, and $\beta(\mathrm{d})$ coefficients.

is that the bead spends more energy deforming the bed because it touches more and more beads, and/or because more frictional contacts are activated. It is consistent with the fact that for big impactors (100 times bigger than the beads constituting the bed), the crater depth and diameter increase with the impact bead diameter. ${ }^{27,28,42}$ The coefficient $\beta$ has a more complex behavior: it starts to decrease from $2.2-2.3$ to $1.5-1.9$ between the smooth and the rough surface, and then oscillates between 2 and 5 . The first fall of the curve for the rough surface may be due to an experimental artifact: as the drops have been performed by increasing the impact velocity, the highest one could have damaged the stuck beads of the surface. The result is a lower energy transfer efficiency to the plate and thus a lower $W_{e l}$ dependency on $V_{z}$. But globally, $\beta$ tends to increase as the bed thickness increases, implying that the elastic energy is more and more sensitive to the velocity. It is not consistent with the previous explanation in terms of impact crater sizes because the crater dimensions increase with the impact velocity $V_{z}$ as well. So more energy is also spent to deform the bed. A possible solution is to distinguish the crater depth $h_{c}$ and diameter $D_{c}$. Indeed, if both correspond to a bed deformation, the first one changes the attenuation experienced by the emitted acoustic wave by modifying the granular thickness crossed. So there is a competition between the energy gain coming from a lower attenuation and the losses coming from a bigger bed deformation. The ratio $h_{c} / D_{c}=\left(V_{z}^{2} / R\right)^{1 / 12}$ shows that an increase of the impact velocity will increase the penetration depth faster than the crater diameter. ${ }^{27}$ And the results seem to show that it is fast enough for lower attenuation to win against the bed deformation.

In conclusion, the scaling law proposed by Farin et al. ${ }^{17}$ from the Hertz theory reproduces experimental results for smooth and rough surfaces, and its adaptation to erodible surfaces seems relevant when the parameters are tuned.

\section{CONCLUSION}

We have shown that it is possible to describe grain impacts on an erodible bed by tuning the parameters of the Hertz scaling law $W_{e l}=W_{e l}^{0} R_{b}^{\alpha} V_{z}^{\beta}\left(R^{2}>0.5\right)$. For an impact on smooth surface, the experimental coefficients $\alpha=5 \pm 0.02$ and $\beta=2.25 \pm 0.05$ are very close to the theoretical values with less than $0.4 \%$ and $2.3 \%$ difference, respectively. $\alpha$ decreases by a factor $\sim 1.12$ and $\beta$ globally increases by a factor $\sim 1.5$ between the smooth surface and the granular bed made of 10 layers of beads. It signifies that the elastic energy is less and less sensitive to the impacting bead radius but more and more to the impact velocity. Furthermore, an exponential decay $W_{e l} / E_{c}^{\text {impact }}=0.13 e^{-0.44 e^{*}}$ of the radiated elastic energy with respect to the bed thickness has been found. It corresponds to an attenuation of a factor 100 between the smooth and the thickest surface, and is compatible with field observations. ${ }^{16,17}$ Moreover, we have shown that the fluctuations of the acoustic energy $W_{e l}$ are much higher for impacts on erodible surfaces than on smooth surfaces, jumping from $\sim 1 \%$ to $\sim 70 \%$. For the rough surface more specifically, we have quantified the effect of the bounce angle of the impacting particle on the impact fluctuations on the radiated elastic energy. The energy amplitude is shown to vary with the bounce angle $\theta$ as $\cos ^{21 / 5}(\theta / 2)$. Despite the complexity of the physical processes involved in bead impacts on erodible beds and of the generated seismic waves, our experiments quantify the effect of an erodible surface on seismic efficiency and source fluctuations. However, the scaling laws may be different on field because rocks are much heavier (about 4 orders of magnitude for rocks of $10 \mathrm{~cm}$ diameter compared to glass beads) and with irregular surface contours. Consequently, the local curvature of the impacted zone differs from the average radius, and plasticity may change the energy balance. Thus, this work is a first step to help interpretation of field measurements of rock impacts and of the associated seismic signal.

\section{ACKNOWLEDGMENTS}

This work has been funded by the ERC Contract No. ERC-CG-2013-PE10-617472 SLIDEQUAKES. We thank J. Laurent and P. Gondret for fruitful discussions.

\footnotetext{
${ }^{1}$ A. Volkwein, K. Schellenberg, V. Labiouse, F. Agliardi, F. Berger, F. Bourrier, L. K. A. Dorren, W. Gerber, and M. Jaboyedoff, Nat. Hazards Earth Syst. Sci. 11, 2617 (2011).

${ }^{2}$ P. Lacroix and A. Helmstetter, Bull. Seismol. Soc. Am. 101, 341 (2011).

${ }^{3}$ V. L. Zimmer and N. Sitar, Eng. Geol. 193, 49 (2015).

${ }^{4}$ F. Dammeier, A. Guilhem, J. R. Moore, F. Haslinger, and S. Loew, Bull. Seismol. Soc. Am. 105, 3001 (2015).

${ }^{5}$ F. Dammeier, J. R. Moore, C. Hammer, F. Haslinger, and S. Loew, J. Geophys. Res.: Earth Surf. 121, 351-371, https://doi.org/10.1002/ 2015JF003647 (2016).

${ }^{6}$ C. Hibert, J.-P. Malet, F. Bourrier, F. Provost, F. Berger, P. Bornemann, P. Tardif, and E. Mermin, Earth Surf. Dyn. 5, 283 (2017).

${ }^{7}$ V. L. Zimmer, B. D. Collins, G. M. Stock, and N. Sitar, Earth Surf. Processes Landforms 37, 680 (2012).

${ }^{8}$ P. Bottelin, D. Jongmans, D. Daudon, A. Mathy, A. Helmstetter, V. Bonilla-Sierra, H. Cadet, D. Amitrano, V. Richefeu, L. Lorier et al., Nat. Hazards Earth Syst. Sci. 14, 3175 (2014).

${ }^{9}$ I. Vilajosana, E. Suriñach, A. Abellán, G. Khazaradze, D. Garcia, and J. Llosa, Nat. Hazards Earth Syst. Sci. 8, 805 (2008).

${ }^{10}$ J. Deparis, D. Jongmans, F. Cotton, L. Baillet, F. Thouvenot, and D. Hantz, Bull. Seismol. Soc. Am. 98, 1781 (2008).
} 
${ }^{11}$ F. Dammeier, J. R. Moore, F. Haslinger, and S. Loew, J. Geophys. Res.: Earth Surf. 116, F04024, https://doi.org/10.1029/2011JF002037 (2011).

${ }^{12}$ C. Hibert, A. Mangeney, G. Grandjean, and N. M. Shapiro, J. Geophys. Res.: Earth Surf. 116, F04032, https://doi.org/10.1029/2011JF002038 (2011).

${ }^{13}$ C. Hibert, A. Mangeney, G. Grandjean, C. Baillard, D. Rivet, N. M. Shapiro, C. Satriano, A. Maggi, P. Boissier, V. Ferrazzini, and W. Crawford, J. Geophys. Res.: Earth Surf. 119, 1082, https://doi.org/ 10.1002/2013JF002970 (2014).

${ }^{14}$ A. Manconi, M. Picozzi, V. Coviello, F. De Santis, and L. Elia, Geophys Res. Lett. 43, 6960-6967, https://doi.org/10.1002/2016GL069572 (2016).

${ }^{15}$ C. Hibert, A. Mangeney, G. Grandjean, A. Peltier, A. DiMuro, N. M. Shapiro, V. Ferrazzini, P. Boissier, V. Durand, and P. Kowalski, J. Volcanol. Geotherm. Res. 333-334, 36 (2017).

${ }^{16}$ J. W. Kean, J. A. Coe, V. Coviello, J. B. Smith, S. W. McCoy, and M. Arattano, Geophys. Res. Lett. 42, 6365, https://doi.org/10.1002/ 2015GL064811 (2015).

${ }^{17}$ M. Farin, A. Mangeney, R. Toussaint, J. D. Rosny, N. Shapiro, T. Dewez, C. Hibert, C. Mathon, O. Sedan, and F. Berger, J. Geophys. Res.: Solid Earth 120, 7102, https://doi.org/10.1002/2015JB012331 (2015).

${ }^{18}$ D. Buttle and C. Scruby, Wear 137, 63 (1990).

${ }^{19}$ D. Buttle, S. Martin, and C. Scruby, J. Res. Nondestr. Eval. 3, 1 (1991).

${ }^{20}$ G. C. McLaskey and S. D. Glaser, J. Acoust. Soc. Am. 128, 1087 (2010).

${ }^{21}$ M. Farin, A. Mangeney, J. de Rosny, R. Toussaint, J. Sainte-Marie, and N. M. Shapiro, J. Sound Vib. 362, 176 (2016).

${ }^{22}$ H. Hertz, J. Angew. Math. 92, 156 (1882).

${ }^{23}$ K. L. Johnson and K. L. Johnson, Contact Mechanics (Cambridge University Press, 1987).

${ }^{24}$ V. C. Tsai, B. Minchew, M. P. Lamb, and J.-P. Ampuero, Geophys. Res. Lett. 39, 102404, https://doi.org/10.1029/2011GL050255 (2012).
${ }^{25}$ J. Barrière, A. Krein, A. Oth, and R. Schenkluhn, Earth Surf. Processes Landforms 40, 913 (2015).

${ }^{26}$ D. L. Roth, E. E. Brodsky, N. J. Finnegan, D. Rickenmann, J. M. Turowski, and A. Badoux, J. Geophys. Res.: Earth Surf. 121, 725, https:// doi.org/10.1002/2015JF003782 (2016).

${ }^{27}$ J. S. Uehara, M. A. Ambroso, R. P. Ojha, and D. J. Durian, Phys. Rev. Lett. 90, 194301 (2003).

${ }^{28}$ A. Seguin, Y. Bertho, and P. Gondret, Phys. Rev. E 78, 010301 (2008).

${ }^{29}$ D. Beladjine, M. Ammi, L. Oger, and A. Valance, Phys. Rev. E 75, 061305 (2007).

${ }^{30}$ M. Ammi, L. Oger, D. Beladjine, and A. Valance, Phys. Rev. E 79, 021305 (2009).

${ }^{31}$ A. Valance and J. Crassous, Eur. Phys. J. E 30, 43 (2009).

${ }^{32}$ F. Bourrier, F. Nicot, and F. Darve, Granular Matter 10, 415 (2008).

${ }^{33}$ A. H. Clark, L. Kondic, and R. P. Behringer, Phys. Rev. Lett. 109, 238302 (2012).

${ }^{34}$ A. H. Clark, A. J. Petersen, L. Kondic, and R. P. Behringer, Phys. Rev. Lett. 114, 144502 (2015).

${ }^{35}$ G. Michlmayr, D. Cohen, and D. Or, Earth-Sci. Rev. 112, 97 (2012).

${ }^{36}$ C. Zener, Phys. Rev. 59, 669 (1941).

${ }^{37}$ H. Goyder and R. White, J. Sound Vib. 68, 59 (1980).

${ }^{38}$ B. W. Drinkwater, M. Castaings, and B. Hosten, J. Acoust. Soc. Am. 113, 3161 (2003)

${ }^{39}$ A. Balvantín, A. Baltazar, and J. I. Aranda-Sanchez, Int. J. Mech. Sci. 63, 66 (2012)

${ }^{40}$ D. Royer and E. Dieulesaint, Elastic Waves in Solids I: Free and Guided Propagation (Springer, Berlin Heidelberg, 2000).

${ }^{41}$ S. W. McCoy, G. E. Tucker, J. W. Kean, and J. A. Coe, J. Geophys. Res.: Earth Surf. 118, 589, https://doi.org/10.1002/jgrf.20041 (2013).

${ }^{42}$ M. A. Ambroso, C. R. Santore, A. R. Abate, and D. J. Durian, Phys. Rev. E 71, 051305 (2005). 